\title{
Hope in the Modern Plague
}

AIDS has been called the Modern Plague, and with more than 58 million people infected with HIV since the beginning of the epidemic, and 22 million of those already dead, that description cannot be termed sensationalist. Despite the intensive global AIDS research effort, which has yielded great advances in understanding the pathogenesis of the disease and some truly efficacious antiretroviral drugs, progress towards an effective preventive vaccine has been frustratingly slow. But the recent Keystone Symposia on HIV provided wary cause for hope in the fight against the global AIDS epidemic. In addition to presentations outlining several promising vaccine candidates, there was tangible evidence that concerted global efforts by various organizations were being geared up to streamline the process of implementing trials in humans. These advances are encouraging signs that a long and arduous research effort may finally be coming to fruition.

At least three of the vaccine candidates that were described demonstrated good protection against challenge virus in monkey models. All three were distinguished by the magnitude and breadth of the cytotoxic T lymphocyte (CTL) responses that they elicited, resulting in marked suppression of viremia and preservation of $\mathrm{CD}^{+} \mathrm{T}$ cell counts. Previous vaccine initiatives had focused on eliciting a neutralizing antibody response, which has proven intractable, and this switch to vaccines that evoke robust CTL responses has been driven by an increasing realization that these cells are the dominant controllers of viral replication. This new approach has benefitted from the convergence of interesting findings from various fields and major improvements in both the vectors and the immunoassay technology.

Some fundamental scientific parameters remain to be defined. What will be the immune correlates of a successful vaccine trial? What will be acceptable clinical end-points? What degree of immunity is the final goal? These questions will be answered and reframed as clinical trials progress and 'more is better' will be a guiding principle, but some goalposts should be set. Sterilizing immunity is now widely accepted to be an unattainable target, and efforts are being directed towards some level of protective immunity as is acceptable in other disease paradigms: a vaccine that doesn't abrogate infection but ameliorates disease.

At least three major challenges now present themselves. First, the perennial problem of how to induce a protective neutralizing antibody response needs to be solved. A vaccination regimen that induced neutralizing antibodies in addition to a strong, broad CTL response would indeed be a potent weapon against HIV, and there needs to be an intensification of research in this area. Secondly, there needs to be a concerted effort to conduct comparative studies to elucidate the most effective combinations of vector, DNA/protein components, route of administration and adjuvant protocols; at least three of the current promising crop of vaccine candidates have come from such comparative studies, some outside of HIV research. And finally, dare it be said, there must be preparations for eventual success: bullding adequate production facilities and providing the infrastructure to deliver inocula to individuals in developing countries are not trivial concerns.

This emergence of promising vaccine candidates has spurred a more aggressive promotion and streamlining of global coordination of trials from bodies such as National Institute for Allergic and Infectious Diseases (NIAID), the International AIDS Vaccine Initiative (IAVI) and a welcome return to the fray for pharmaceutical companies in the form of Merck and their capital muscle. It is also heartening that vaccines derived from HIV clades that predominate in developing countries are now being developed - just last month, the first AIDS vaccine candidate specifically designed for Africa began in Kenya. The ramifications will be manifest, deflecting charges of 'scientific colonialism', building good faith within developing countries and hopefully leading to concerted efforts to improve existing infrastructures.

Towards this last aim, recent initiatives to provide effective antiretrovirals to developing countries at affordable prices should be applauded. There has been some criticism that treatment initiatives will not be effective due to the patient compliance requirements and that such efforts will divert funding and attention from preventive efforts. This inapproprate dichotomization of treatment and prevention is unwelcome: they are not mutually exclusive options and an integrated program of the two must be pursued. In order to build the infrastructures that will be required once an effective vaccine is available, trust must be won, and the provision of concrete help now will help foster those good relations. Treatment modalities may very well be synergistic with prevention efforts since lower viral load translates to lowered vertical transmission, and there is evidence that it also impacts on horizontal transmission. There is also, of course, a compelling moral case that the industrialized nations cannot deny treatments that have proven so effective at prolonging life.

We are now poised at the point of testing in humans some promising and potentially efficacious vaccines. There remains a long journey ahead that must be tempered with appropriate restraint, but the future already looks a little brighter. 